\title{
A Human Blueprint for Al Coexistence
}

\author{
Kai-Fu Lee
}

\section{Contents}

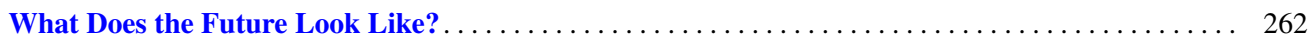

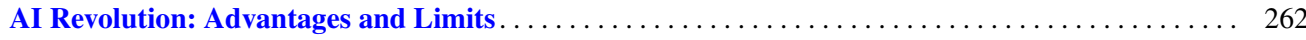

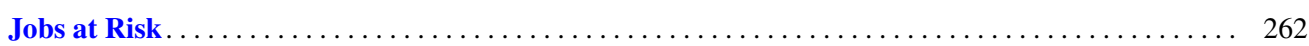

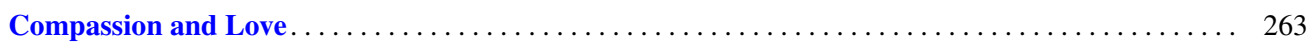

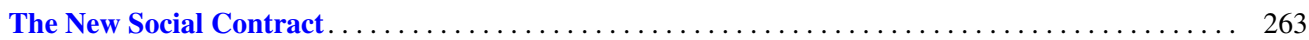

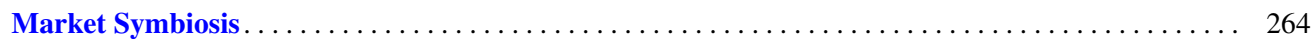

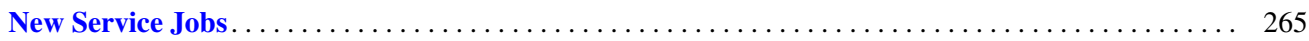

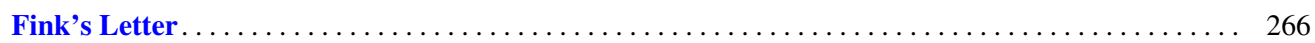

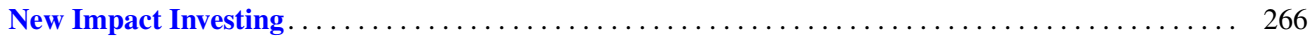

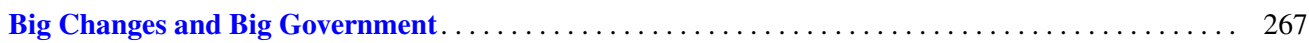

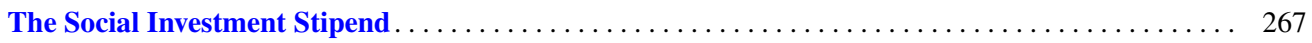

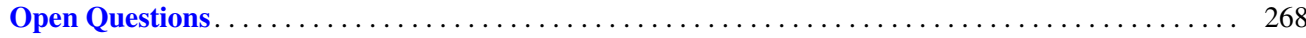

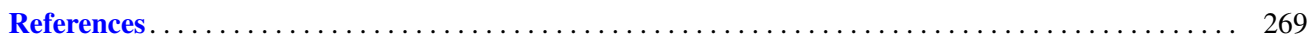

\section{Abstract}

The positive coexistence of humans and AI is possible and needs to be designed as a system that provides for all members of society, but one that also uses the wealth generated by AI to build a society that is more compassionate, loving, and ultimately human. It is incumbent on us to use the economic abundance of the AI age to foster the values of volunteers who devote their time and energy toward making their communities more caring. As a practical measure, to protect against AI/robotics' labor saving and job displacement effects, a "social investment stipend" should be explored. The stipend would be given to those who invest their time and energy in those activities that promote a kind, compassionate, and creative society, i.e., care work, community service, and education. It would put the economic bounty generated by AI to work in building a better society, rather than just numbing the pain of AIinduced job losses.

\section{Keywords}

Artificial intelligence $\cdot$ Robotics $\cdot$ Employment . Income $\cdot$ Social investment $\cdot$ Compassion

K.-F. Lee $(\varangle)$

Sinovation Ventures, Beijing, China

e-mail:kfl@chuangxin.com

J. von Braun et al. (eds.), Robotics, AI, and Humanity, https://doi.org/10.1007/978-3-030-54173-6_22 


\section{What Does the Future Look Like? ${ }^{1}$}

Artificial intelligence is a technology that sparks the human imagination. What will our future look like as we come to share the earth with intelligent machines? Utopians believe that once AI far surpasses human intelligence, it will provide us with near-magical tools for alleviating suffering and realizing human potential. In this vision, super-intelligent AI systems will so deeply understand the universe that they will act as omnipotent oracles, answering humanity's most vexing questions and conjuring brilliant solutions to problems such as disease and climate change.

But not everyone is so optimistic. The best-known member of the dystopian camp is the technology entrepreneur Elon Musk, who has called super-intelligent AI systems "the biggest risk we face as a civilization," comparing their creation to "summoning the demon" (Kumparak 2014). This group warns that when humans create self-improving AI programs whose intellect dwarfs our own, we will lose the ability to understand or control them. Which vision to accept? I'd say neither.

They simply aren't possible based on the technology we have today or any breakthroughs that might be around the corner. Both scenarios would require "artificial general intelligence"- that is, AI systems that can handle the incredible diversity of tasks done by the human brain. Making this jump would require several fundamental scientific breakthroughs, each of which may take many decades.

\section{Al Revolution: Advantages and Limits}

The AI revolution will be of the magnitude of the Industrial Revolution - but probably larger and definitely faster. Where the steam engine only took over physical labor, AI can perform both intellectual and physical labor. And where the Industrial Revolution took centuries to spread beyond Europe and the USA, AI applications are already being adopted simultaneously all across the world.

AI's main advantage over humans lies in its ability to detect incredibly subtle patterns within large quantities of data and to learn from them. While a human mortgage officer will look at only a few relatively crude measures when deciding whether to grant you a loan (your credit score, income and age), an AI algorithm will learn from thousands of lesser variables (what web browser you use, how often you buy groceries, etc.). Taken alone, the predictive power of each of these is minuscule, but added together, they yield a

\footnotetext{
${ }^{1}$ This chapter is adapted from the author's book AI Superpowers: China, Silicon Valley and the New World Order (2018a) and the corresponding website at https://aisuperpowers.com as well as the author's article in The Wall Street Journal dating from the same year (Lee 2018b).
}

far more accurate prediction than the most discerning people are capable of.

For cognitive tasks, this ability to learn means that computers are no longer limited to simply carrying out a rote set of instructions written by humans. Instead, they can continuously learn from new data and perform better than their human programmers. For physical tasks, robots are no longer limited to repeating one set of actions (automation) but instead can chart new paths based on the visual and sensor data they take in (autonomy).

Together, this allows AI to take over countless tasks across society: driving a car, diagnosing a disease, or providing customer support. AI's superhuman performance of these tasks will lead to massive increases in productivity. According to a June 2017 study by the consulting firm PwC, AI's advance will generate $\$ 15.7$ trillion in additional wealth for the world by 2030 (PwC 2017). This is great news for those with access to large amounts of capital and data. It's very bad news for anyone who earns their living doing soon-to-be-replaced jobs.

There are, however, limits to the abilities of today's AI, and those limits hint at a hopeful path forward. While AI is great at optimizing for a highly narrow objective, it is unable to choose its own goals or to think creatively. And while $\mathrm{AI}$ is superhuman in the coldblooded world of numbers and data, it lacks social skills or empathy-the ability to make another person feel understood and cared for. Analogously, in the world of robotics, AI is able to handle many crude tasks like stocking goods or driving cars, but it lacks the delicate dexterity needed to care for an elderly person or infant.

\section{Jobs at Risk}

What does that mean for workers who fear being replaced? Jobs that are asocial and repetitive, such as fast-food preparers or insurance adjusters, are likely to be taken over in their entirety. For jobs that are repetitive but social, such as bartenders and doctors, many of the core tasks will be done by AI, but there remains an interactive component that people will continue to perform. The jobs that will be safe, at least for now, are those well beyond the reach of AI's capabilities in terms of creativity, strategy, and sociability, from social workers to CEOs.

Even where AI doesn't destroy jobs outright, however, it will exacerbate inequality. AI is inherently monopolistic: A company with more data and better algorithms will gain ever more users and data. This self-reinforcing cycle will lead to winner-take-all markets, with one company making massive profits while its rivals languish.

A similar consolidation will occur across professions. The jobs that will remain relatively insulated from AI fall on op- 
posite ends of the income spectrum. CEOs, home care nurses, attorneys, and hairstylists are all in "safe" professions, but the people in some of these professions will be swimming in the riches of the AI revolution while others compete against a vast pool of desperate fellow workers.

We can't know the precise shape and speed of AI's impact on jobs, but the broader picture is clear. This will not be the normal churn of capitalism's creative destruction, a process that inevitably arrives at a new equilibrium of more jobs, higher wages, and better quality of life for all.

Many of the free market's self-correcting mechanisms will break down in an AI economy. The twenty-first century may bring a new caste system, split into a plutocratic AI elite and the powerless struggling masses.

Recent history has shown us just how fragile our political institutions and social fabric can be in the face of disruptive change. If we allow AI economics to run their natural course, the geopolitical tumult of recent years will look like child's play.

On a personal and psychological level, the wounds could be even deeper. Society has trained most of us to tie our personal worth to the pursuit of work and success. In the coming years, people will watch algorithms and robots easily outmaneuver them at tasks they've spent a lifetime mastering. I fear that this will lead to a crushing feeling of futility and obsolescence. At worst, it will lead people to question their own worth and what it means to be human.

\section{Compassion and Love}

But in developing a blueprint for human coexistence with AI, we need to remember that intelligent machines will increasingly be able to do our jobs and meet our material needs, disrupting industries and displacing workers in the process. But there remains one thing that only human beings are able to create and share with one another: love.

With all of the advances in machine learning, the truth remains that we are still nowhere near creating AI machines that feel any emotions at all. Can you imagine the elation that comes from beating a world champion at the game you've devoted your whole life to mastering? AlphaGo, the first computer Go program to beat a human professional Go player, did just that, but it took no pleasure in its success, felt no happiness from winning, and had no desire to hug a loved one after its victory. Despite what science-fiction films like Her-in which a man and his artificially intelligent computer operating system fall in love-portray, AI has no ability or desire to love or be loved.

It is in this uniquely human potential for growth, compassion, and love where I see hope. I believe we must forge a new synergy between artificial intelligence and the human heart, and look for ways to use the forthcoming material abundance generated by artificial intelligence to foster love and compassion in our societies.

If we can do these things, I believe there is a path toward a future of both economic prosperity and spiritual flourishing. Navigating that path will be tricky, but if we are able to unite behind this common goal, I believe humans will not just survive in the age of AI. We will thrive like never before.

\section{The New Social Contract}

The challenges before us remain immense. Within 15 years, I predict that we will technically be able to automate 40$50 \%$ of all jobs in the United States. That does not mean all of those jobs will disappear overnight, but if the markets are left to their own devices, we will begin to see massive pressure on working people. China and other developing countries may differ slightly in the timing of those impacts, lagging or leading in job losses depending on the structures of their economies. But the overarching trend remains the same: rising unemployment and widening inequality.

Techno-optimists will point to history, citing the Industrial Revolution and the nineteenth-century textile industry as "proof" that things always work out for the best. But as we've seen, this argument stands on increasingly shaky ground. The coming scale, pace, and skill-bias of the AI revolution mean that we face a new and historically unique challenge. Even if the most dire predictions of unemployment do not materialize, AI will take the growing wealth inequality of the internet age and accelerate it tremendously.

We are already witnessing the way that stagnant wages and growing inequality can lead to political instability and even violence. As AI rolls out across our economies and societies, we risk aggravating and quickening these trends. Labor markets have a way of balancing themselves out in the long run, but getting to that promised long run requires we first pass through a trial by fire of job losses and growing inequality that threaten to derail the process. Meeting these challenges means we cannot afford to passively react. We must proactively seize the opportunity that the material wealth of AI will grant us and use it to reconstruct our economies and rewrite our social contracts. The epiphanies that emerged from my experience with cancer were deeply personal, but I believe they also gave me a new clarity and vision for how we can approach these problems together.

Building societies that thrive in the age of AI will require substantial changes to our economy but also a shift in culture and values. Centuries of living within the industrial economy have conditioned many of us to believe that our primary role in society (and even our identity) is found in productive, wage-earning work. Take that away and you have broken one of the strongest bonds between a person and his or her community. As we transition from the industrial age to the 
AI age, we will need to move away from a mindset that equates work with life or treats humans as variables in a grand productivity optimization algorithm. Instead, we must move toward a new culture that values human love, service, and compassion more than ever before.

No economic or social policy can "brute force" a change in our hearts. But in choosing different policies, we can reward different behaviors and start to nudge our culture in different directions. We can choose a purely technocratic approachone that sees each of us as a set of financial and material needs to be satisfied - and simply transfer enough cash to all people so that they don't starve or go homeless. In fact, this notion of universal basic income (UBI) seems to be becoming more and more popular these days (Ito 2018). But in making that choice I believe we would both devalue our own humanity and miss out on an unparalleled opportunity. Instead, I want to lay out proposals for how we can use the economic bounty created by AI to double-down on what makes us human. Doing this will require rewriting our fundamental social contracts and restructuring economic incentives to reward socially productive activities in the same way that the industrial economy rewarded economically productive activities.

\section{Market Symbiosis}

The private sector is leading the AI revolution, and, in my mind, it must also take the lead in creating the new, more humanistic jobs that power it. Some of these will emerge through the natural functioning of the free market, while others will require conscious efforts by those motivated to make a difference.

Many of the jobs created by the free market will grow out of a natural symbiosis between humans and machines. While AI handles the routine optimization tasks, human beings will bring the personal, creative, and compassionate touch. This will involve the redefinition of existing occupations or the creation of entirely new professions in which people team up with machines to deliver services that are both highly efficient and eminently human. AI will do the analytical thinking, while humans will wrap that analysis in warmth and compassion.

A clear example of human-AI symbiosis for the upperleft-hand quadrant can be found in the field of medicine. I have little doubt that $\mathrm{AI}$ algorithms will eventually far surpass human doctors in their ability to diagnose disease and recommend treatments. Legacy institutions-medical schools, professional associations, and hospitals - may slow down the adoption of these diagnostic tools, using them only in narrow fields or strictly as reference tools. But in a matter of a few decades, I'm confidentthat the accuracy and efficiency gains will be so great that AI-driven diagnoses will take over eventually.

One response to this would be to get rid of doctors entirely, replacing them with machines that take in symptoms and spit out diagnoses. But patients don't want to be treated by a machine, a black box of medical knowledge that delivers a cold pronouncement: "You have fourth-stage lymphoma and a 70 percent likelihood of dying within five years." Instead, patients will desire - and I believe the market will create-a more humanistic approach to medicine.

Traditional doctors could instead evolve into a new profession, one that I'll call a "compassionate caregiver." These medical professionals would combine the skills of a nurse, medical technician, social worker, and even psychologist. Compassionate caregivers would be trained not just in operating and understanding the diagnostic tools but also in communicating with patients, consoling them in times of trauma, and emotionally supporting them throughout their treatment. Instead of simply informing patients of their objectively optimized chances of survival, they could share encouraging stories, saying "Kai-Fu had the same lymphoma as you and he survived, so I believe you can too."

These compassionate caregivers would not compete with machines in their ability to memorize facts or optimize treatment regimens. In the long run that's a losing battle. Compassionate caregivers would be well trained, but in activities requiring more emotional intelligence, not as mere vessels for the canon of medical knowledge. They would form a perfect complement to the machine, giving patients unparalleled accuracy in their diagnoses as well as the human touch that is so often missing from our hospitals today. In this humanmachine symbiosis created by the free market, we would inch our society ahead in a direction of being a little kinder and a little more loving.

Best of all, the emergence of compassionate caregivers would dramatically increase both the number of jobs and the total amount of medical care given. Today, the scarcity of trained doctors drives up the cost of healthcare and drives down the amount of quality care delivered around the world. Under current conditions of supply and demand, it's simply not cost-feasible to increase the number of doctors. As a result, we strictly ration the care they deliver. No one wants to go wait in line for hours just to have a few minutes with a doctor, meaning that most people only go to hospitals when they feel it's absolutely necessary. While compassionate caregivers will be well trained, they can be drawn from a larger pool of workers than doctors and won't need to undergo the years of rote memorization that is required of doctors today. As a result, society will be able to costeffectively support far more compassionate caregivers than there are doctors, and we would receive far more and better care. 


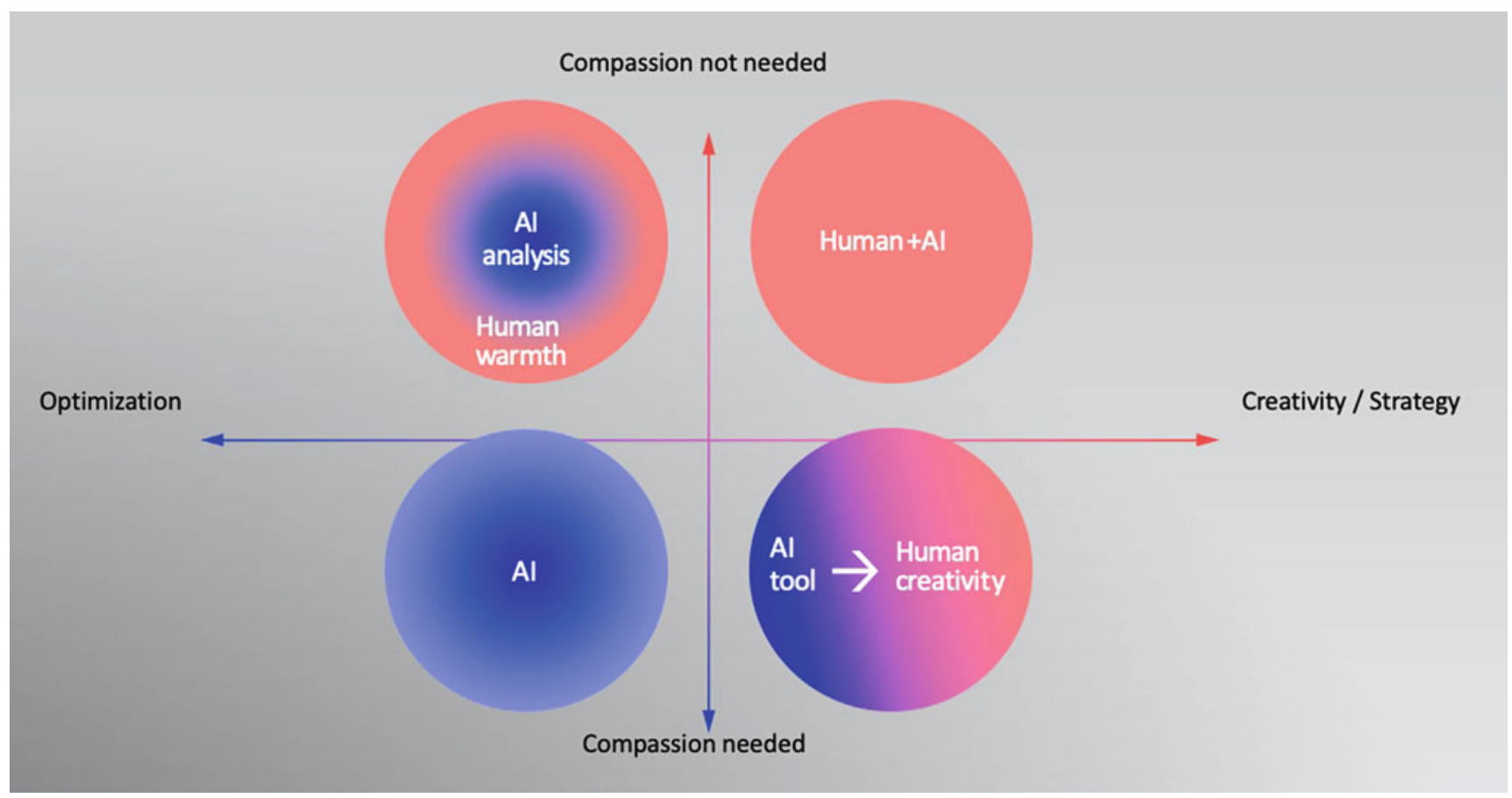

Human-AI coexistence in the labor market. Source: Lee (2018b: 211)

Similar synergies will emerge in many other fields: teaching, law, event planning, and high-end retail. Paralegals at law firms could hand their routine research tasks off to algorithms and instead focus on communicating more with clients and making them feel cared for. AI-powered supermarkets like the Amazon Go store may not need cashiers anymore, so they could greatly upgrade the customer experience by hiring friendly concierges.

For those in professional sectors, it will be imperative that they adopt and learn to leverage AI tools as they arrive. As with any technological revolution, many workers will find the new tools both imperfect in their uses and potentially threatening in their implications. But these tools will only improve with time, and those who seek to compete against AI on its own terms will lose out. In the long run, resistance may be futile, but symbiosis will be rewarded.

Finally, the internet-enabled sharing economy will contribute significantly to alleviating job losses and redefining work for the AI age. We'll see more people step out of traditional careers that are being taken over by algorithms, instead using new platforms that apply the "Uber model" to a variety of services. We see this already in Care.com, ${ }^{2}$ an online platform for connecting caregivers and customers, and I believe we will see a blossoming of analogous models in education and other fields. Many mass-market goods and services will be captured by data and optimized by algorithms, but some of the more piecemealor personalized work within

\footnotetext{
${ }^{2}$ https://en.wikipedia.org/wiki/Care.com
}

the sharing economy will remain the exclusive domain of humans.

In the past, this type of work was constrained by the bureaucratic costs of running a vertical company that attracted customers, dispatched workers, and kept everyone on the payroll even when there wasn't work to be done. The platformatization of these industries dramatically increases their efficiency, increasing total demand and take-home pay for the service workers themselves. Adding AI to the equationas ride-hailing companies like Didi and Uber have already done-will only further boost efficiency and attract more workers.

\section{New Service Jobs}

Beyond the established roles in the sharing economy, I'm confident we will see entirely new service jobs emerge that we can hardly imagine today. Explain to someone in the 1950s what a "life coach" was and they'd probably think you were goofy. Likewise, as AI frees up our time, creative entrepreneurs and ordinary people will leverage these platforms to create new kinds of jobs. Perhaps people will hire "season changers" who redecorate their closets every few months, scenting them with flowers and aromas that match the mood of the season. Or environmentally conscious families will hire "home sustainability consultants" to meet with the family and explore creative and fun ways for the household to reduce its environmental footprint. 
But despite all these new possibilities created by profitseeking businesses, I' $m$ afraid the operations of the free market alone will not be enough to offset the massive job losses and gaping inequality on the horizon. Private companies already create plenty of human-centered service jobs-they just don't pay well. Economic incentives, public policies, and cultural dispositions have meant that many of the most compassion-filled professions existing today often lack job security or basic dignity.

The U.S. Bureau of Labor Statistics has found that home health aides and personal care aides are the two fastest growing professions in the country (Casselman 2017), with an expected growth of 1.2 million jobs by 2026. But annual income in these professions averages just over $\$ 20,000$ (U.S. Department of Labor 2018, 2019). Other humanistic labors of love-stay-at-home parenting, caring for aging or disabled relatives-aren't even considered a "job" and receive no formal compensation.

exactly the kinds of loving and compassionate activities that we should embrace in the AI economy, but the private sector has proven inadequate so far at fostering them. There may come a day when we enjoy such material abundance that economic incentives are no longer needed. But in our present economic and cultural moment, money still talks. Orchestrating a true shift in culture will require not just creating these jobs but turning them into true careers with respectable pay and greater dignity.

Encouraging and rewarding these prosocial activities means going beyond the market symbiosis of the private sector. We will need to re-energize these industries through service sector impact investing and government policies that nudge forward a broader shift in cultural values.

\section{Fink's Letter}

When a man overseeing \$5.7 trillion speaks, the global business community tends to listen. So when BlackRock founder Larry Fink, head of the world's largest asset management company, posted a letter to CEOs demanding greater attention to social impact, it sent shockwaves through corporations around the globe. In the letter, titled "A Sense of Purpose," Fink (2018) wrote, "We . . . see many governments failing to prepare for the future, on issues ranging from retirement and infrastructure to automation and worker retraining. As a result, society increasingly is turning to the private sector and asking that companies respond to broader societal challenges. ... Society is demanding that companies, both public and private, serve a social purpose. . . . Companies must benefit all of their stakeholders, including shareholders, employees, customers, and the communities in which they operate." Fink's letter dropped just days before the 2018 World Economic Forum, an annual gathering of the global financial elite in Davos, Switzerland. I was attending the forum and watched as CEOs anxiously discussed the stern warning from a man whose firm controlled substantial ownership stakes in their companies. Many publicly professed sympathy for Fink's message but privately declared his emphasis on broader social welfare to be anathema to the logic of private enterprise.

Looked at narrowly enough, they're right: publicly traded companies are in it to win it, bound by fiduciary duties to maximize profits. But in the age of AI, this cold logic of dollars and cents simply can't hold. Blindly pursuing profits without any thought to social impact won't just be morally dubious; it will be downright dangerous.

Fink referenced automation and job retraining multiple times in his letter. As an investor with interests spanning the full breadth of the global economy, he sees that dealing with AI-induced displacement is not something that can be left entirely up to free markets. Instead, it is imperative that we reimagine and reinvigorate corporate social responsibility, impact investing, and social entrepreneurship.

In the past, these were the kinds of things that business people merely dabbled in when they had time and money to spare. Sure, they think, why not throw some money into a microfinance startup or buy some corporate carbon offsets so we can put out a happy press release touting it. But in the age of AI, we will need to seriously deepen our commitment to-and broaden our definition of - these activities. Whereas these have previously focused on feel-good philanthropic issues like environmental protection and poverty alleviation, social impact in the age of AI must also take on a new dimension: the creation of large numbers of service jobs for displaced workers.

\section{New Impact Investing}

As a venture-capital investor, I see a particularly strong role for a new kind of impact investing. I foresee a venture ecosystem emerging that views the creation of humanistic service-sector jobs as a good in and of itself. It will steer money into human-focused service projects that can scale up and hire large numbers of people: lactation consultants for postnatal care, trained coaches for youth sports, gatherers of family oral histories, nature guides at national parks, or conversation partners for the elderly. Jobs like these can be meaningful on both a societal and personal level, and many of them have the potential to generate real revenue-just not the $10,000 \%$ returns that come from investing in a unicorn technology startup.

Kick-starting this ecosystem will require a shift in mentality for venture capitalists (VCs) who participate. The very idea of venture capital has been built around high risks and exponential returns. When an investor puts money into ten 
startups, they know full well that nine of them most likely will fail. But if that one success story turns into a billion-dollar company, the exponential returns on that one investment make the fund a huge success. Driving those exponential returns are the unique economics of the internet. Digital products can be scaled up infinitely with near-zero marginal costs, meaning the most successful companies achieve astronomical profits.

Service-focused impact investing, however, will need to be different. It will need to accept linear returns when coupled with meaningful job creation. That's because human-driven service jobs simply cannot achieve these exponential returns on investment. When someone builds a great company around human care work, they cannot digitally replicate these services and blast them out across the globe. Instead, the business must be built piece by piece, worker by worker. The truth is, traditional VCs wouldn't bother with these kinds of linear companies, but these companies will be a key pillar in building an AI economy that creates new jobs and fosters human connections.

There will of course be failures, and returns will never match pure technology $\mathrm{VC}$ funds. But that should be fine with those involved. The ecosystem will likely be staffed by older VC executives who are looking to make a difference, or possibly by younger VC types who are taking a "sabbatical" or doing "pro bono" work. They will bring along their keen instincts for picking entrepreneurs and building companies, and will put them to work on these linear service companies. The money behind the funds will likely come from governments looking to efficiently generate new jobs, as well as companies doing corporate social responsibility.

Together, these players will create a unique ecosystem that is much more jobs-focused than pure philanthropy, much more impact-focused than pure venture capital. If we can pull together these different strands of socially conscious business, I believe we'll be able to weave a new kind of employment safety net, all while building communities that foster love and compassion.

In this respect I am most excited about the impact of AI on healthcare and education. These two sectors are ready for AI disruption and can deploy AI for good. For example, we have invested in a company that uses AI and big data to optimize supply chains, reducing medication shortages for over 150 million people living in rural China. We also have drug discovery companies combining deep learning and generative chemistry to shorten drug discovery time by a factor of three to four. In education, we see companies developing AI solutions to improve English pronunciation, grade exams, and homework, and personalize and gamify math learning. This will free teachers from routine tasks, and allow them to spend time building inspirational and stimulating connections and with our next generations.

\section{Big Changes and Big Government}

And yet, for all the power of the private market and the good intentions of social entrepreneurs, many people will still fall through the cracks. We need look no further than the gaping inequality and destitute poverty in so much of the world today to recognize that markets and moral imperatives are not enough. Orchestrating a fundamental change in economic structures often requires the full force of governmental power. If we hope to write a new social contract for the age of AI, we will need to pull on the levers of public policy.

There are some in Silicon Valley who see this as the point where UBI comes into play. Faced with inadequate job growth, the government must provide a blanket guarantee of economic security, a cash transfer that can save displaced workers from destitution and which will also save the tech elite from having to do anything else about it.

The unconditional nature of the transfer fits with the highly individualistic, live-and-let-live libertarianism that undergirds much of Silicon Valley. Who is the government, UBI proponents ask, to tell people how to spend their time? Just give them the money and let them figure it out on their own. It's an approach that matches how the tech elite tend to view society as a whole. Looking outward from Silicon Valley, they often see the world in terms of "users" rather than citizens, customers rather than members of a community. I have a different vision. I don't want to live in a society divided into technological castes, where the AI elite live in a cloistered world of almost unimaginable wealth, relying on minimal handouts to keep the unemployed masses sedate in their place. I want to create a system that provides for all members of society, but one that also uses the wealth generated by AI to build a society that is more compassionate, loving, and ultimately human.

\section{The Social Investment Stipend}

Just as those volunteers devoted their time and energy toward making their communities a little bit more loving, I believe it is incumbent on us to use the economic abundance of the AI age to foster the values of volunteers who devote their time and energy toward making their communities a little bit more loving and encourage this same kind of activity. To do this, I propose we explore the creation not of a UBI but of what I call a social investment stipend. The stipend would be a decent government salary given to those who invest their time and energy in those activities that promote a kind, compassionate, and creative society. These would include three broad categories: care work, community service, and education. 
These would form the pillars of a new social contract, one that valued and rewarded socially beneficial activities in the same way we currently reward economically productive activities. The stipend would not substitute for a social safety net-the traditional welfare, healthcare, or unemployment benefits to meet basic needs-but would offer a respectable income to those who choose to invest energy in these socially productive activities. Today, social status is still largely tied to income and career advancement. Endowing these professions with respect will require paying them a respectable salary and offering the opportunity for advancement like a normal career. If executed well, the social investment stipend would nudge our culture in a more compassionate direction. It would put the economic bounty of AI to work in building a better society, rather than just numbing the pain of AIinduced job losses.

Each of the three recognized categories-care, service, and education-would encompass a wide range of activities, with different levels of compensation for full- and part-time participation. Care work could include parenting of young children, attending to an aging parent, assisting a friend or family member dealing with illness, or helping someone with mental or physical disabilities live life to the fullest. This category would create a veritable army of people-loved ones, friends, or even strangers-who could assist those in need, offering them what my entrepreneur friend's touchscreen device for the elderly never could: human warmth. Service work would be similarly broadly defined, encompassing much of the current work of nonprofit groups as well as volunteers. Tasks could include performing environmental remediation, leading afterschool programs, guiding tours at national parks, or collecting oral histories from elders in our communities. Participants in these programs would register with an established group and commit to a certain number of hours of service work to meet the requirements of the stipend.

Finally, education could range from professional training for the jobs of the AI age to taking classes that could transform a hobby into a career. Some recipients of the stipend will use that financial freedom to pursue a degree in machine learning and use it to find a high-paying job. Others will use that same freedom to take acting classes or study digital marketing.

Bear in mind that requiring participation in one of the above activities is not something designed to dictate the daily activities of each person receiving the stipend. That is, the beauty of human beings lies in our diversity, the way we each bring different backgrounds, skills, interests, and eccentricities. I don't seek to smother that diversity with a command-and-control system of redistribution that rewards only a narrow range of socially approved activities. But by requiring some social contribution in order to receive the stipend, we would foster a far different ideology than the laissez-faire individualism of a UBI. Providing a stipend in exchange for participation in prosocial activities reinforces a clear message: It took efforts from people all across society to help us reach this point of economic abundance. We are now collectively using that abundance to recommit ourselves to one another, reinforcing the bonds of compassion and love that make us human.

Looking across all the activities above, I believe there will be a wide enough range of choices to offer something suitable to all workers who have been displaced by AI. The more people-oriented may opt for care work, the more ambitious can enroll in job-training programs, and those inspired by a social cause may take up service or advocacy jobs. In an age in which intelligent machines have supplanted us as the cogs and gears in the engine of our economy, I hope that we will value all of these pursuits-care, service, and personal cultivation-as part of our collective social project of building a more human society.

\section{Open Questions}

Implementing a social investment stipend will of course raise new questions and frictions: How much should the stipend be? Should we reward people differently based on their performance in these activities? How do we know if someone is dutifully performing their "care" work? And what kinds of activities should count as "service" work? These are admittedly difficult questions, ones for which there are no clear-cut answers. Administering a social investment stipend in countries with hundreds of millions of people will involve lots of paperwork and legwork by governments and the organizations that create these new roles.

But these challenges are far from insurmountable. Governments in developed societies already attend to a dizzying array of bureaucratic tasks just to maintain public services, education systems, and social safety nets. Our governments already do the work of inspecting buildings, accrediting schools, offering unemployment benefits, monitoring sanitary conditions at hundreds of thousands of restaurants, and providing health insurance to tens of millions of people. Operating a social investment stipend would add to this workload, but I believe it would be more than manageable. Given the huge human upside to providing such a stipend, I believe the added organizational challenges will be well worth the rewards to our communities. But what about affordability? Offering a living salary to people performing all of the above tasks would require massive amounts of revenue, totals that today appear unworkable in many heavily indebted countries. AI will certainly increase productivity across society, but can it really generate the huge sums necessary to finance such dramatic expansion in government expenditures?

This too remains an open question, one that will only be settled once the AI technologies themselves proliferate 
across our economies. If AI meets or exceeds predictions for productivity gains and wealth creation, I believe we could fund these types of programs through super taxes on super profits. Yes, it would somewhat cut into economic incentives to advance AI, but given the dizzying profits that will accrue to the winners in the AI age, I don't see this as a substantial impediment to innovation. But it will take years to get to that place of astronomical profits, years during which working people will be hurting. To smooth the transition, I propose a slow ratcheting up of assistance. While leaping straight into the full social investment stipend described above likely won't work, I do think we will be able to implement incremental policies along the way. These piecemeal policies could both counteract job displacement as it happens and move us toward the new social contract articulated above.

We could start by greatly increasing government support for new parents so that they have the choice to remain at home or send their child to full-time daycare. For parents who choose to home-school their kids, the government could offer subsidies equivalent to a teacher's pay for those who attain certain certifications. In the public school systems, the number of teachers could also be greatly expandedpotentially by a factor as high as ten-with each teacher tasked with a smaller number of students that they can teach in concert with AI education programs. Government subsidies and stipends could also go to workers undergoing job retraining and people caring for aging parents. These simple programs would allow us to put in place the first building blocks of a stipend, beginning the work of shifting the culture and laying the groundwork for further expansion.

As AI continues to generate both economic value and worker displacement, we could slowly expand the purview of these subsidies to activities beyond care work or job training. And once the full impact of AI-very good for productivity, very bad for employment-becomes clear, we should be able to muster the resources and public will to implement programs akin to the social investment stipend. When we do, I hope that this will not just alleviate the economic, social, and psychological suffering of the AI age. Rather, I hope that it will further empower us to live in a way that honors our humanity and empowers us to do what no machine can: share our love with those around us.

\section{References}

Casselman, B. (2017). A peek at future jobs reveals growing economic divides. Available via New York Times. Retrieved February 27, 2020, from https://www.nytimes.com/2017/10/24/business/ economy/future-jobs.html

Fink, L. (2018). Larry Fink's annual letter to CEOs: A sense of purpose. Available via BlackRock. Retrieved February 27, 2020, from https://www.blackrock.com/corporate/en-us/investor-relations/ larry-fink-ceo-letter

Ito, J. (2018). The paradox of universal basic income. Available via WIRED. Retrieved February 27, 2020, from https://www.wired.com/ story/the-paradox-of-universal-basic-income/

Kumparak, G. (2014). Elon Musk compares building artificial intelligence to "summoning the demon". Available via TC. Retrieved February 27, 2020, from https://techcrunch.com/2014/10/26/elonmusk-compares-building-artificial-intelligence-to-summoning-thedemon/

Lee, K.-F. (2018a). AI superpowers: China, Silicon Valley, and the new world order. New York: Houghton Mifflin Harcourt.

Lee, K.-F. (2018b, September 14). The human promise of the AI revolution. The Wall Street Journal. https://www.wsj.com/articles/ the-human-promise-of-the-ai-revolution-1536935115

PwC. (2017). Sizing the price: PwC's global artificial intelligence study: Exploiting the AI revolution. Available via pwc. Retrieved February 27, 2020, from https://www.pwc.com/gx/en/issues/dataand-analytics/publications/artificial-intelligence-study.html

U.S. Department of Labor, Bureau of Labor Statistics, Occupational Employment Statistics. (2018). Personal care aides. Available via U.S. Bureau of Labor Statistics. Retrieved February 27, 2020, from https://www.bls.gov/oes/current/oes399021.htm

U.S. Department of Labor, Bureau of Labor Statistics, Occupational Employment Statistics. (2019). Home health aides and personal care aides. Available via U.S. Bureau of Labor Statistics. Retrieved February 27, 2020, from https://www.bls.gov/ooh/healthcare/homehealth-aides-and-personal-care-aides.htm

Open Access This chapter is licensed under the terms of the Creative Commons Attribution 4.0 International License (http://creativecommons. $\mathrm{org} /$ licenses/by/4.0/), which permits use, sharing, adaptation, distribution and reproduction in any medium or format, as long as you give appropriate credit to the original author(s) and the source, provide a link to the Creative Commons license and indicate if changes were made.

The images or other third party material in this chapter are included in the chapter's Creative Commons license, unless indicated otherwise in a credit line to the material. If material is not included in the chapter's Creative Commons license and your intended use is not permitted by statutory regulation or exceeds the permitted use, you will need to obtain permission directly from the copyright holder. 